Editorial

\title{
Acknowledgement to Reviewers of Molbank in 2013
}

Molbank Editorial Office, MDPI AG, Klybeckstrasse 64, CH-4057 Basel, Switzerland

Published: 26 February 2014

The editors of Molbank would like to express their sincere gratitude to the following reviewers for assessing manuscripts in 2013:

Abonía, Rodrigo
Abuqamar, Synan F.
Bonacorso, Helio G.
Cheng, Yixiang
Chimichi, Stefano
Coelho, Paulo
Dehaen, Wim
Hormi, Osmo E. O.
Friestad, Gregory K.
González, Javier
Gubica, Tomasz
Haider, Norbert
Ishchenko, Alexander A.
Jarosz-Wilkolazka, Anna
Kacprzak, Karol
Kielland, Nicola
Kitson, Sean L.
Kleij, Arjan W.
Kobatake, Seiya
Kurteva, Vanya
La Rosa, Concetta
Lee, Sunwoo

Litinas, Konstantinos
Lu, Ping
Mahmoodi, Nosrat O.
Marinkovic, A.D.
Menezes, Paulo H.
Meng, Ge
Mruthyunjayaswamy, B. H. M.
Navarro-Vázquez, Armando
Nechifor, Marioara
Odell, Luke R.
Okamoto, Noriko
Ośmiałowski, Borys
Paleta, Oldřich
Panday, Sharad Kumar
Pankajakshan, Sreekumar
Miranda, Paulo C. M. L.
Pinho e Melo, Teresa M. V. D.
Ponticelli, Fabio
Raposo, M. Manuela M.
Rasapalli, Sirappa
Refat, Moamen S.
Savic, Vladimir

Seijas, Julio A.

Shi, Da-Qing

Silveira, Claudio C.

Sloop, Joseph

Sukhorukov, Alexey Yu.

Sun, Wei

Takizawa, Shinobu

Thiemann, Thies

Towns, Andrew

Tran, Thanh-Dao

Urabe, Hirokazu

Vázquez-López, Ezequiel M.

Yamaguchi, Tadatsugu

Yamamoto, Yoshinori

Yamanaka, Masahiro

Yang, Jye-Shane

Yavari, Issa

Zarei, Maaroof

Zonta, Cristiano

(C) 2014 by the authors; licensee MDPI, Basel, Switzerland. This article is an open access article distributed under the terms and conditions of the Creative Commons Attribution license (http://creativecommons.org/licenses/by/3.0/). 